\title{
Les élites africaines et la langue française : une appropriation controversée
}

Alice Goheneix

\section{OpenEdition}

1 Journals

Édition électronique

URL : https://journals.openedition.org/dhfles/117

DOI : $10.4000 /$ dhfles. 117

ISSN : 2221-4038

Éditeur

Société Internationale pour l'Histoire du Français Langue Étrangère ou Seconde

Édition imprimée

Date de publication : 1 janvier 2008

Pagination : 133-150

ISSN : 0992-7654

Référence électronique

Alice Goheneix, «Les élites africaines et la langue française : une appropriation controversée »,

Documents pour l'histoire du français langue étrangère ou seconde [En ligne], 40/41 | 2008, mis en ligne le 17 décembre 2010, consulté le 27 mai 2021. URL : http://journals.openedition.org/dhfles/117 ; DOI : https://doi.org/10.4000/dhfles.117

Ce document a été généré automatiquement le 27 mai 2021.

(C) SIHFLES 


\title{
Les élites africaines et la langue française : une appropriation controversée
}

\author{
Alice Goheneix
}

\section{Introduction}

1 Vingt ans après la parution de Orientalism ${ }^{2}$, la question postcoloniale semble avoir émergé en France. En effet, depuis la célébration en 1998 du $150^{\mathrm{e}}$ anniversaire de l'abolition de l'esclavage dans les colonies françaises, les crispations françaises autour $\mathrm{du}$ passé colonial se sont multipliées ${ }^{3}$, remettant en question le mythe de l'universalisme républicain: ce récit partiellement réel et partiellement imaginaire, constitué du réseau de références politiques implicites et explicites qui font de la République française la « patrie » des droits de l'homme; cette croyance française selon laquelle la République serait l'incarnation constitutionnelle d'un pur nationalisme civique ne pouvant que mettre en œuvre les vertus d'égalité et de fraternité proclamées par sa devise.

2 Alors même que quelques philosophes et universitaires français - comme Derrida, Foucault, Balandier - avaient contribué à fonder les approches méthodologiques de ce qui deviendrait la théorie postcoloniale et que, parallèlement, des écrivains de langue française anciennement colonisés (dont Césaire, Senghor, Fanon) avaient posé au cœur de leurs réflexions quelques-unes de ses problématiques centrales, on semble (re)découvrir cette pensée en France après les années 2000 ; comme en témoigne la véritable explosion ces toutes dernières années d'ouvrages et de numéros spéciaux, de périodiques et de traductions $s^{4}$. En conséquence, le mythe de l'universalisme républicain est soupçonné d'avoir masqué dans la société française le maintien de pratiques discriminatoires et de représentations héritées de la colonisation.

3 Ce questionnement s'est clairement organisé autour de la loi du 23 février en 2005. Dans son article 4 (abrogé depuis par voie réglementaire), cette loi stipulait : 
Les programmes de recherche universitaire accordent à l'histoire de la présence française outre-mer, notamment en Afrique du Nord, la place qu'elle mérite. Les programmes scolaires reconnaissent en particulier le rôle positif de la présence française outre-mer, notamment en Afrique du Nord [...].

4 Cet article réhabilitait ainsi le "bien-fondé » et les soi-disant résultats de la mission civilisatrice, dans la mesure où les députés français scindaient la période coloniale en deux moments. Le premier moment, la guerre de conquête, est reconnu comme violent et condamnable ${ }^{5}$; tandis que le second, celui de la «mise en valeur», de la «mission civilisatrice ", reste fondamentalement mémorisé par les élus comme modernisateur ${ }^{6}$.

Or, parmi les « effets positifs » de la colonisation, l'enseignement de la langue française et en langue française constituait une référence implicite du texte de loi ${ }^{7}$. Les polémiques - mémorielles et institutionnelles, militantes et universitaires - autour de la loi du 23 février, et plus généralement autour d'un questionnement français postcolonial, ont donc assez logiquement fait ressurgir le vieux procès intenté à la Francophonie ${ }^{8}$, selon lequel elle constituerait à la fois une trace de l'empire colonial français et servirait toujours les intérêts de l'ancienne métropole.

6 Cette inquiétude, cet inconfort à l'égard de la politique française de la langue, m'étaient déjà apparus clairement lors d'une série d'entretiens que j'avais effectués en 2005 auprès d'une quarantaine d'expatriés français en Asie du Sud-Est; mais ils prenaient une dimension publique en mars 2007 avec le Manifeste de 44 écrivains francophones. Ces derniers, dans un texte signé et publié dans le journal Le Monde le 16 mars 2007, critiquaient frontalement les institutions francophones comme étant toujours fondées sur « un pacte exclusif avec la nation ». Ces écrivains disaient leur souffrance et leur colère d'être systématiquement considérés comme des périphériques et revendiquaient une littérature en français polycentrée et reconnaissant l'égale dignité créative de tous ses membres. Parallèlement à la controverse suscitée par ce manifeste, il semble qu'une très grande majorité de publications traitant de l'histoire et de la défense de la francophonie se doit de prendre position sur ce sujet, afin de la défendre d'une accusation de néo-colonialisme ou d'entreprise impériale.

7 Il semble que ce malaise dans la francophonie repose sur un a priori: compte tenu du passif colonial de la langue française, le bannissement de cette langue, en tant que symbole de l'oppression, aurait dû constituer la règle plutôt que l'exception dans les colonies. Or les anciens colonisés ont largement conservé et revendiqué l'usage du français.

Dès lors, comment expliquer cette pérennité du français comme langue officielle dans l'ancienne AOF et l'ancienne AEF?

9 Dans une première partie, nous reviendrons sur le récit classique qui est fait de l'histoire de la Francophonie et de son appropriation par les anciens colonisés. Nous essaierons ensuite de démontrer en quoi ce récit mythistorique ${ }^{9}$ tend à masquer les lignes de fond (sociologiques, politiques et économiques) qui peuvent expliquer le maintien du français comme langue officielle dans les anciennes colonies africaines. Enfin, dans une dernière partie, nous soulignerons les conséquences problématiques de cette appropriation pour la position française à l'égard de la francophonie. 


\section{La mythistoire francophone}

10 À partir des publications de l'Organisation internationale de la Francophonie ${ }^{10}$ et de celles d'auteurs et d'universitaires défendant les institutions francophones ${ }^{11}$, il est possible de reconstituer un récit unique, une histoire officielle de la francophonie. Cette histoire est construite à partir de faits réels, mais aussi d'impasses historiques (ou en tout cas d'une absence de questions) et repose sur deux personnages clefs : Onésime Reclus et Léopold Sédar Senghor. Ce récit, partiellement mythologique et partiellement historique, pourrait ainsi être qualifié de récit «mythistorique $»^{12}$. Ce récit fonctionne en deux temps ${ }^{13}$ : le premier temps est colonial, c'est celui d'Onésime Reclus. Le deuxième temps est postcolonial (au sens strictement chronologique ici), c'est celui de Léopold Sédar Senghor.

11 Dans l'ensemble des publications sur la " francophonie ", il existe en effet un consensus selon lequel Onésime Reclus a inventé le terme dans les années 1880, dans un livre intitulé France, Algérie et Colonies ${ }^{14}$ (publié en 1886 et qui a pu l'être une première fois en 1880). Dans ce livre, Reclus définit la francophonie à la fois comme le monde des "parlant-français ", mais également comme une communauté politique. À la lumière de ses autres publications ${ }^{15}$, il apparaît en effet convaincu que «la langue fait les peuples » et contribue à transformer leurs modes de pensée et leurs valeurs. Dans les prises de position sur Onésime Reclus, le présupposé raciste de ses travaux est souvent évité, parfois démenti. On trouve ainsi sur le site de $L a$ Documentation française, à propos des travaux de Reclus: "C'est un hymne à la conquête coloniale que compose le géographe, concevant une véritable doctrine de l'impérialisme française. Mais sa conception du colonialisme ne s'appuie pas sur des considérations mercantilistes ou raciales; son argumentation est géographique, linguistique, démographique. ${ }^{16}$ » Une telle affirmation tend à laisser penser que Reclus avait une argumentation objective, scientifique, basée sur des critères indiscutables et irréprochables. En réalité, l'argumentation "géographique, linguistique, démographique » de Reclus s'inscrit directement dans la lignée des discours et théories nationalistes et colonialistes du XIX ${ }^{\mathrm{e}}$ siècle. En ce sens, Reclus est un héritier de Jules Duval, Prévost Paradol ou ChasseloupLaubat, les pères de la mission civilisatrice ${ }^{17}$ ferryste ${ }^{18}$. Quant au racisme, il faut lire les écrits de Reclus pour se convaincre que la hiérarchisation des peuples et de leur(s) langue(s) constituait bien un des présupposés de la « nécessaire » expansion française.

12 L'argumentation de Reclus est en réalité triple. D'une part elle s'appuie sur une obsession de la décadence française (essentiellement pour des raisons démographiques); ensuite sur la guerre coloniale que se livrent les puissances européennes ; enfin sur l'idée que certains peuples seront plus perméables à la pensée française. Dans l'esprit de Reclus, il s'agit bien de convertir les peuples colonisés à l'esprit français. De créer de l'identique, du «même », pour assurer la pérennité de la nation et du génie français. On est donc face à une pensée ambivalente, qui repose sur des présupposés racialistes en même temps qu'une ambition assimilationniste, que Walzer qualifierait d'universaliste dogmatique ${ }^{19}$. De fait, Reclus constitue une référence bien embarrassante pour l'idée contemporaine de francophonie.

Cette naissance irrévocablement nationaliste peut donc expliquer l'insistance qui est faite, dans le récit consensuel de la francophonie multilatérale, sur le deuxième moment fondateur, novembre 1962. Cette date, celle de la publication du numéro intitulé «Le français, langue vivante » de la revue Esprit, est en effet présentée comme 
la véritable naissance de la véritable francophonie. Ses défenseurs veulent ainsi prouver que ce sont bien les anciens colonisés, africains et asiatiques - et non pas l'ancienne métropole - qui décidèrent de faire de la langue française l'objet et le sujet d'une organisation internationale. Ainsi, il est systématiquement rappelé que ce sont des héros des indépendances africaines et asiatiques (on comptait parmi les auteurs Habib Bourguiba, Hamani Diori, Norodom Sihanouk), proclamant à la fois leur attachement à la langue et leurs identités culturelles qui créèrent la francophonie.

14 S'appuyant sur le fait que le mot francophonie est en définitive très peu usité entre 1880 et 1960, les défenseurs de la francophonie considèrent 1962 comme la naissance de la véritable francophonie. Cette renaissance, qui est effectivement à l'origine d'une francophonie institutionnelle multilatérale ${ }^{20}$, conforte donc les observateurs de la Francophonie dans l'idée que l'accusation d'impérialisme ou de néocolonialisme est une vue de l'esprit, une "idée reçue ", pour reprendre le titre de l'ouvrage de Claire Tréan $^{21}$. Luttant contre les accusations de néo-colonialisme et d'impérialisme français culturel et linguistique, les défenseurs de la francophonie insistent sur le fait que la francophonie n'est pas seulement une langue en partage (simple outil de communication), mais une communauté de valeurs. Ces valeurs sont supposées être celles de la francité senghorienne, en définitive celle de l'universalisme abstrait des Lumières, émancipateur et respectueux des identités plurielles... celles de l'universalisme républicain.

L'histoire consensuelle de la Francophonie constitue donc clairement une entreprise de légitimation. L'appropriation et la défense par les dirigeants africains de la langue de l'ancien colonisateur disculperait ainsi la langue française de visées nationalistes et néocolonialistes françaises.

Paradoxalement, les historiens et politistes qui s'inscrivent dans le courant des études postcoloniales contribuent dans une certaine mesure à légitimer cette histoire officielle de l'appropriation de la langue par les dirigeants africains. Dans la théorie postcoloniale en effet, le terme « appropriation » est

[...] utilisé pour décrire les façons par lesquelles les sociétés postcoloniales s'emparent de certains aspects de la culture impériale - langue, tournures d'écriture, films, théâtre, l'ensemble des modes de pensée et d'argumentation tels que le rationalisme, la logique et l'analyse - susceptibles de leur être utiles dans l'articulation de leurs propres identités sociales et culturelles. [...] La théorie postcoloniale se focalise sur l'exploration des manières par lesquelles la culture dominée ou colonisée peut utiliser les outils du discours dominant pour résister à son contrôle politique ou culturel ${ }^{22}$.

Selon cette théorie, les anciens colonisés qui maitrisent la langue sont à même de l'utiliser pour la faire parler contre le processus de colonisation. En en modifiant les formes discursives et en l'utilisant pour exprimer leurs propres expériences historiques, sociales ou psychiques, les anciens colonisés peuvent en faire un instrument de libération. Ce processus a également l'avantage de permettre aux anciens colonisés de toucher une audience beaucoup plus large qu'avec l'usage de leur seule langue maternelle. Cette position repose sur le postulat selon lequel la langue elle-même ne constitue pas une aliénation irrévocable et peut très bien être utilisée pour exprimer une pensée anticoloniale. Ce concept d'appropriation est ainsi souvent associé à celui d'abrogation, qui traduit le rejet d'une structure standardisée de la langue par le colonisateur. 

laquelle la langue (du colonisateur) véhicule des paradigmes dominants immuables :

L'abrogation implique plutôt que la maison du maître est toujours adaptable et que les mêmes outils offrent un moyen de transformation conceptuelle et de libération ${ }^{23}$.

19 Bien que cette théorisation s'appuie sur la conservation de la langue anglaise ${ }^{24}$ comme langue officielle et sur son appropriation par les écrivains des anciennes colonies britanniques, les écrivains de langue française de la Négritude peuvent s'inscrire dans cette conception émancipatrice de l'appropriation de la langue française. À cet égard, l'œuvre d'Aimé Césaire, et en particulier le Discours sur le colonialisme, constituerait une très explicite illustration de cette subversion du colonialisme dans la langue du colonisateur ${ }^{25}$.

En définitive, la renaissance de la francophonie en 1962 rapprochée de la théorie postcoloniale peut nous conduire à penser que la francophonie constitue une entreprise d'appropriation réussie, en tant qu'elle prend le nationalisme linguistique du colonisateur à son propre piège. En effet, en se saisissant de la langue française pour proclamer leurs spécificités culturelles, les colonisés participent d'une déconnexion de la langue et de sa nation d'origine. La nation française perd l'exclusivité de la maitrise et de l'attachement à sa langue; tandis que la langue française devient susceptible d'exprimer d'autres expériences nationales. En outre, en dénonçant le colonialisme dans la langue du colonisateur, les anciens colonisés délégitiment le fondement prétendument civilisateur de son expansion. En conséquence, d'une part la présence de la langue française à l'étranger ne dépend plus de la seule initiative française, d'autre part n'exprime plus la seule civilisation française. En d'autres termes, la francophonie aurait bel et bien rompu « le pacte exclusif de la langue avec la nation », et le manifeste sur la littérature-monde de mars $2007^{26}$ ne constituerait qu'un ultime soubresaut d'une entreprise initiée il y a 45 ans.

\section{L'histoire interstitielle de la francophonie : ce que le récit officiel ne dit pas}

21 Néanmoins, cette argumentation reste discutable pour plusieurs raisons. La première est qu'elle ne fait pas l'économie de la problématique inhérente à la théorie postcoloniale. En effet, soutenir la légitimité de la francophonie par le choix des anciens colonisés, n'est-ce pas prolonger ad vitam aeternam une lecture positionnelle de la francophonie ? Positionnelle, c'est-à-dire toujours relative à la position de dominant ou de dominé pendant la période coloniale. Loin de réaliser une communauté politique idéale fondée sur l'égalité des membres, la francophonie reposerait intrinsèquement sur une alliance entre anciens inégaux; sa légitimité actuelle resterait fondée sur la revanche linguistique des colonisés. Ce besoin systématique de légitimation par l'argumentation postcoloniale maintiendrait en définitive la francophonie (comme communauté linguistique et comme organisation multilatérale) dans des structures relatives au colonialisme et resterait impensable en dehors de lui. Cette idée peut nous permettre de mieux entendre la dénonciation des 44 écrivains du Manifeste :

Soyons clairs: l'émergence d'une littérature-monde en langue française consciemment affirmée, ouverte sur le monde, transnationale, signe l'acte de décès 
de la francophonie. Personne ne parle le francophone, ni n'écrit en francophone. La

francophonie est de la lumière d'étoile morte. Seconde Guerre mondiale ne visait pas l'assimilation culturelle et politique, c'est-à-dire la formation de citoyens français de plein droit dans les colonies. L'éducation s'inscrivait dans les projets de mise en valeur et visait avant tout à former des auxiliaires efficaces pour l'administration française. Elle visait à former des sujets de l'Empire; pas des citoyens français. La lecture de Georges Hardy, inspecteur de l'enseignement en $\mathrm{AOF}$ est à cet égard très éclairante ${ }^{27}$ : selon lui, compte tenu des risques que recèle la maîtrise de la langue et la formation de haut niveau (susceptible de transformer les masses des colonisés en classes dangereuses), la très grande majorité des colonisés ne doit être formée qu'à une maîtrise de la langue usuelle, fonctionnelle. La plénitude de la langue ne doit, elle, n'être réservée qu'à une " élite indigène ». Cette élite était en effet supposée d'autant plus réceptive à la culture d'expression française que la métropole pourrait lui assurer un statut socio-économique privilégié. La langue contribuait ainsi à créer de l'attachement politique et à faire comprendre aux colonisés le « bien-fondé » de la mission civilisatrice.

Les élites politiques africaines qui vont revendiquer la défense de la francophonie ont fait partie de ces rares hommes du début $d u X X^{e}$ siècle qui ont commencé ou fait l'intégralité de leurs études en Afrique et les ont continuées en France. Un bref rappel des parcours universitaires des pères africains de la francophonie est à cet égard très instructif : ainsi Léopold Sédar Senghor, premier Africain agrégé de l'université de Paris en 1935, s'engagera dans la vie politique après la Libération et deviendra député du Sénégal à l'Assemblée nationale française en 1945, avant d'être Secrétaire d'État dans le cabinet Edgar Faure (1955-1956), puis ministre-conseiller au début de la V ${ }^{\mathrm{e}}$ République (1959) ; Habib Bourguiba mène des études de droit et de sciences politiques à Paris et devient avocat avant de retourner en Tunisie en 1927 et de s'engager dans la presse indépendantiste et de fonder en 1934 le " Néo-Destour »; quant à Hamani Diori, qui fondera le "Parti progressiste nigérien» (PPN), et sera élu député du Niger à l'Assemblée nationale française en 1946 - dont il deviendra vice-président en 1957 - il a fait ses études à Dakar avant de devenir instituteur dans une des Écoles normales mises en place par la métropole ${ }^{28}$. 

France comme la «nation dont le génie est comme destiné à élever pas à pas les hommes vers les sommets de la dignité et de la fraternité où tous pourront s'unir un jour ». Cette conférence des hauts fonctionnaires, convoquée à Brazzaville sous l'influence de Félix Éboué, ouvre donc clairement la participation «des indigènes à leurs propres affaires ", puisqu'elle vise à doter les territoires coloniaux d'assemblées et de les faire représenter à l'Assemblée constituante de l'Union française. Après les annonces de Brazzaville, les représentants des colonies étaient en droit d'attendre l'octroi d'une citoyenneté pleine et entière au sein de l'Union française. La loi Lamine Gueye du 7 mai 1946 allait dans ce sens, puisqu'elle accordait la citoyenneté française à tous les ressortissants des territoires d'outre-mer. En revanche, la Constitution d'octobre 1946 restait relativement ambiguë puisqu'elle juxtaposait une idée fédérale d'égalité entre les collectivités nationales ( «la France forme avec les peuples d'outremer une Union fondée sur l'égalité des droits et des devoirs ») et une conception très assimilatrice de l'égalité des hommes « sans distinction de race ni de religion ».

Ainsi, à la fin des années 1940 et dans le courant des années 1950, pour bon nombre de représentants des colonies, la revendication de l'indépendance n'allait pas de soi ; et une partie d'entre eux a donc défendu des projets pleinement intégrationnistes ou fédéraux. D'autant que ces dirigeants ont tous conscience que l'autonomie, voire l'indépendance sans les moyens de l'indépendance sera difficile; qu'il faut continuer à développer les territoires, à promouvoir l'éducation de masse, à former des cadres. Ainsi Senghor a dénoncé le choix entre statut de département et statut d'État associé proposé par la constitution de 1946. Selon lui en effet, le statut d'État associé intégrait insuffisamment les territoires africains à la République française, tandis que la départementalisation faisait courir le risque d'une assimilation passive, sans métissage ni respect des langues et des cultures autochtones. En 1953, il se prononce donc pour «une République fédérale française »; cette conception va ensuite évoluer vers l'idée d'un Commonwealth à la française - appuyée par Bourguiba dans les années 1960 - puis vers l'idée d'une communauté franco-africaine. 

très proches de ceux de la Francophonie actuelle : «En construisant l'Union française nous pouvons réaliser le plus grand ensemble humain de l'avenir ${ }^{29}$. " Convaincu que "l'indépendance politique sans l'indépendance économique n'avait aucun sens", conscient du temps nécessaire pour que l'Afrique forme ses propres cadres et ses propres élites, Boigny considère le soutien économique et technique français comme un impératif de développement. Il oppose alors à la « mystique de l'indépendance », source d'hostilité et de conflictualité entre anciens colonisateurs et anciens colonisés, « une mystique de la fraternité » :

Nous avons le droit de dire que la véritable indépendance, c'est celle que, sans faiblir, avec la conscience aiguë des intérêts de la jeune et vieille Afrique, nous défendrons de concert avec nos anciens colonisateurs, pour qu'ensemble nous puisions donner à l'univers l'exemple de la vraie fraternité, sans laquelle le monde, malgré toutes ses richesses, malgré tous ses progrès scientifiques, sera appelé à être enseveli définitivement sous les décombres du progrès ${ }^{30}$.

Lorsque le projet de Communauté est rejeté en 1960 au profit d'une accession pacifique à l'indépendance, ces leaders, conscients de la dépendance économique de leurs jeunes États à l'égard de l'ancienne métropole, vont chercher à promouvoir une nouvelle forme de coopération bilatérale et multilatérale qui complète le dispositif français d'aide au développement - qui a commencé de se structurer en 1946, avec la création du FIDES (Fonds d'investissement et de développement économique et social) - et permette de contrer les nostalgiques du cartiérisme ${ }^{31}$. La promotion d'institutions franco-africaines adossées à la langue française résulte donc assez nettement de l'échec du fédéralisme et d'une prise de conscience aiguë des nécessités économiques. Ainsi, Senghor, dans un entretien donné à Jeune Afrique en juin 1966 déclarait :

Pour développer notre économie, nous avons certes besoin de capitaux, mais nous avons surtout besoin d'hommes. [...] C'est la raison pour laquelle d'ailleurs j'en suis venu à la francophonie ; parce que nous avons surtout besoin de cadres formés.

Ainsi, plusieurs projets de coopération économique multilatérale vont être présentés dans les années 1960 par les pays africains, qui lient à la fois l'approche culturelle et le soutien économique. En septembre 1966, Hamani Diori présente un avant-projet d'une communauté mondiale des nations faisant usage du français. Ce projet repose sur trois cercles concentriques dont le premier, qui rassemblait la France et les États africains membres de l'OCAM(l'Organisation commune africaine et malgache) visait très explicitement une coopération culturelle et économique. Ce projet, potentiellement trop coûteux, sera rejeté par la France (qui se serait retrouvée seule pourvoyeuse de fonds). En ce sens, la création de l'Agence de coopération culturelle et technique en 1970 peut légitimement apparaître comme la peau de chagrin d'une idée fédérale et de liens économiques forts entre France et anciennes colonies. Et de fait, cela aura pris du temps, mais l'ACCT aura fini par conduire à un engagement financier français important, puisqu'elle est pérennisée sous le nom d'Agence intergouvernementale de la Francophonie en 1996, puis d'Organisation internationale de la Francophonie en 1998.

\section{Les implications problématiques de l'appropriation sur le nationalisme linguistique français}

Le maintien de la langue française dans les anciennes colonies ne résulte pas d'une volonté politique ex nihilo, mais bien de processus de long terme ancrés dans la

Documents pour l'histoire du français langue étrangère ou seconde, 40/41 | 2008 
sociologie des élites africaines et dans les impératifs politico-économiques de leurs jeunes États. Or, dans le récit officiel de la Francophonie, l'appropriation de la langue par les élites africaines repose sur une conception essentialiste de la langue française qui tend à la sacraliser. À cet égard, les ambiguïtés senghoriennes apparaissent comme exemplaires. Dans le numéro d'Esprit de 1962 déjà cité, Senghor, dans l'article intitulé "Le français, langue de culture", comparait les propriétés des "langues négroafricaines » et celles du français. Selon lui, la langue française incarnerait la clarté, l'intelligibilité et la rationalité. Elle offrirait une capacité accrue de conceptualisation et véhiculerait, plus que les autres, l'humanisme français, "qui exprime toujours la moralité $\aleph^{32}$. Cette argumentation est très ambivalente. Elle semble en effet reposer sur l'analyse objective d'un linguiste, qui veut démontrer l'excellence du système syntaxique et grammatical de la langue française. Mais, ce faisant, elle contribue à conforter le nationalisme linguistique métropolitain. En effet, ces caractéristiques linguistiques, présentées comme spécifiques et intrinsèques au français, sont combinées à une potentialité accrue de conceptualisation, de complexification et d'articulation de la pensée. Dans cet article, le français s'impose à Senghor comme la langue cartésienne par excellence, en même temps qu'elle offre, par le truchement du surréalisme, des potentialités poétiques et sensibles inespérées. Le français se trouve sacralisé à la fois comme langue de la raison et comme langue de la sensibilité.

De fait, ce panégyrique de la langue française, écrit par un homme instruit en français en pleine colonisation, conclut en apothéose le récit hagiographique du nationalisme linguistique français, fondé sur les liens symbiotiques qu'entretiennent la nation et la langue française. Ce récit national conforte l'idée selon laquelle la langue aurait construit la nation et la nation se serait construite dans la langue, par une série d'événements historiques ${ }^{33}$ : le serment de Strasbourg en 842 (par lequel Charles le Chauve et Louis le Germanique s'engagent réciproquement en langues franque et germanique à respecter les frontières de leurs territoires), l'édit de Villers-Cotterêts en 1539 (en vertu duquel les décisions de justice ne seront désormais rendues qu'en français), la création de l'Académie française en 1635, etc. Ce récit s'arrête traditionnellement au traité de Versailles de 1919, qui signifierait, en même temps que la fin de l'exclusivité du français dans les relations diplomatiques, un certain déclin du rayonnement français. Ce récit démontre que toute discussion en France qui se réfère explicitement à la langue se réfère implicitement à la nation française. La langue, en vertu de l'article 2 de la Constitution de 1958, demeure la langue de la République, un lien simultanément civique et émotionnel entre les citoyens français. Elle constitue bien l'une des clefs de voûte mythiques de la nation française. Ainsi Senghor, en concluant son argumentation par l'association «naturelle» de la langue et de l'humanisme français, participe d'une glorification de la langue française, en laissant penser que ne peuvent se transmettre et se dire dans cette langue que les seules valeurs humanistes. Compte tenu de l'association de la langue et de la nation pour la société française, cette glorification de la langue tend à nourrir la sacralisation de la nation française et le mythe français de l'universalisme républicain.

En conséquence, il est possible de proposer une lecture paradoxale, au sens étymologique, des événements francophones de 1962, et susceptible d'expliquer le malaise français à l'égard de la francophonie. Selon cette lecture, la renaissance postcoloniale de la francophonie ne l'aurait pas lavée de son péché colonial originel; au contraire. Le choix des colonisés de conserver la langue française, même s'il est en 
réalité multifactoriel, a pu nourrir les représentations françaises liées à la mission civilisatrice. Le vote de la loi du 23 février 2005, et la polémique qui s'en est ensuivie, ont en effet démontré que les représentations nationalistes héritées de la colonisation ont perduré pour une partie de la société française et ont créé un profond malaise pour une autre partie. À cet égard, la francophonie apparaît comme un sujet extrêmement piégeux.

Les anciens colonisés ont conservé la langue française après la décolonisation pour des raisons diplomatiques, financières et sociales. Les élites africaines qui l'ont défendue gardaient la langue en même temps qu'elles rejetaient l'entreprise coloniale. Mais ce qui leur apparaissait très clair peut ne pas l'avoir été autant pour une société française fondée très largement sur une représentation universaliste et messianique de sa nation et de sa langue. Il nous semble donc possible de formuler l'hypothèse selon laquelle dans la société française, compte tenu de l'étroite imbrication de la langue et de la nation, la conservation de la langue peut avoir été perçue comme une manifestation de gratitude et de reconnaissance des « effets positifs » de la colonisation. En définitive, la nation française, grâce à la transmission de sa langue, aurait accompli sa «mission civilisatrice ${ }^{34} »$; cette langue ayant permis aux peuples colonisés de penser leur indépendance.

39 Cet effet pervers du nationalisme linguistique français pourrait en particulier expliquer le sentiment de malaise que semble ressentir une partie de la société française à l'égard de l'expansion de sa langue outre-mer. La responsabilité en incombe au récit national français lui-même, fondé dans un rapport au monde de type messianique. À cet égard, les polémiques mémorielles identitaires et les prises de position universitaires sur la nécessaire indépendance de l'historiographie vis-à-vis de l'État peuvent apparaître comme autant de fissures dans un récit national paradigmatique qui ne parviendrait plus à défendre son autorité ; un espace se libère peut-être pour un récit national français qui prenne véritablement acte des questions éthiques posées par la colonisation et d'une société française véritablement plurielle.

En conclusion, rappelons la position d'Abdou Diouf: «Le français ne vous appartient plus. Nous l'avons en partage $»^{35}$. Cette phrase énonce clairement la volonté d'émancipation de la francophonie des tentations nationalistes françaises, mais, en même temps, elle met à mal cette pierre fondatrice pour la nation française que constitue la langue. En définitive, l'appropriation de la langue française par les partenaires francophones, si conforme au départ aux aspirations diplomaticoculturelles françaises, aura provoqué un retour de balancier bien difficile à gérer pour la nation.

\section{NOTES}

1. Également doctorante associée à l'IRSE, Institut de recherche stratégique de l'École militaire.

2. Saïd (Edward W.), Orientalism, Penguin Books, 1978. 
3. Bertrand (Romain), Mémoires d'empire, La controverse autour du «fait colonial», Broissieux, Éditions du Croquant, 2006.

4. Cohen (Jim), «La bibliothèque coloniale en pleine expansion", in Mouvements, $\mathrm{n}^{\circ} 51$, septembre 2007 (numéro spécial intitulé : « Qui a peur du postcolonial ? »).

5. Bertrand (Romain), op. cit.

6. Sur cette scission de la période coloniale, cf. Bertrand (Romain), op. cit.

7. Ibid. Cet élément apparaît dans les débats parlementaires qui ont précédé le vote.

8. Classiquement, nous utilisons «Francophonie " avec une majuscule pour nous référer à la "francophonie institutionnelle », c'est-à-dire aux organisations multi-latérales en charge de la promotion internationale de la langue française; l'absence de majuscule correspond à une définition moins stricte, à l'idée d'une communauté transnationale de parlant-français.

9. J'emprunte le terme de mythistoire à Baczko (Bronislav) utilisé dans Lumières de l'Utopie, Paris, Payot, 1978.

10. France, Algérie et colonies, Hachette, 1886.

11. Notamment Guillou (Michel), La francophonie : nouvel enjeu mondial, Paris, Hatier, 1993 ; Léger (Jean-Marc), La francophonie, grand dessein, grande ambiguité, Montréal, Hurtubise, 1987 ; Tréan (Claire), La Francophonie, préface d'Abdou Diouf, éd. Le Cavalier bleu, coll. « Idées reçues ", 2006 ; Tritter (Jean-Louis), Histoire de la langue française, Paris, Ellipses, 1999 ; Saint Robert (Marie-Josée de) ; Wolton (Dominique), Demain la Francophonie, Paris, Flammarion, 2006.

12. Philippe Lavodrama parle de "mythification de l'histoire ou historicisation du mythe", "Senghor et la réinvention du concept de francophonie », in Les Temps Modernes, avril-juillet 2007.

13. Sur l'idée de double généalogie légitimatrice, voir Lavodrama, article cité.

14. Reclus (Onésime), France, Algérie et colonies, Paris, Hachette, 1886.

15. Notamment Le partage du monde, Paris, Librairie universelle, 1906 ; Un grand destin commence, Paris, La Renaissance du Livre, 1917 ; Lâchons l'Asie, prenons l'Afrique : où renaître ? et comment durer ?, Paris, Librairie universelle, 1904 ; La France et ses colonies, Paris, Librairie Hachette et Cie, 1889.

16. Source: <http://www.ladocumentationfrancaise.fr/dossiers/francophonie/onesimereclus.shtml>

17. Ces auteurs sont identifiés par Raoul Girardet comme les leaders des trois foyers qui ont nourri « l'idée coloniale » en France.

18. Les débats parlementaires dans lesquels Ferry défend "le devoir des races supérieures à l'égard des races inférieures " ont été récemment compilés dans Manceron (Gilles), 1885 : le tournant colonial de la République, Jules Ferry contre Georges Clemenceau et autres affrontements parlementaires sur la conquête coloniale, Paris, La Découverte, 2007.

19. Walzer (Michael), « Les deux universalismes », in Esprit, $\mathrm{n}^{\circ}$ 12, décembre 1992.

20. Avec la création de l'Agence de coopération culturelle et technique en 1970, rebaptisée Agence internationale de la Francophonie en 1996 puis Organisation internationale de la Francophonie en 1998.

21. Tréan (Claire), op. cit.

22. Ashcroft (Bill), Griffiths (Gareth), Tiffin (Helen), Postcolonial Studies, The Key Concepts, Londres, Routledge, 2000, p. 19-20; notre traduction.

23. Ibid., p. 5-6; notre traduction.

24. Ashcroft (Bill), Griffiths (Gareth), Tiffin (Helen), The Empire Writes Back, New York, Routledge, 1989.

25. Césaire (Aimé), Discours sur le colonialisme,Paris, éd. Réclames, 1950.

26. "Pour une "littérature-monde" en français, le manifeste de quarante-quatre écrivains en faveur d'une langue française qui serait "libérée de son pacte exclusif avec la nation" ", texte paru dans Le Monde du 16 mars 2007, accessible sur <www.lianes.org/Manifeste-pour-unelitterature-monde-en-français_a128.html>. 
27. Notamment Hardy (Georges), Une conquête morale, l'enseignement en A.O.F., Paris, Armand Colin, 1917.

28. $\quad$ http://www.ladocumentationfrancaise.fr/dossiers/francophonie/peresfrancophonie.shtml>; sur le parcours de Senghor: Dijan (Jean-Michel), Léopold Sédar Senghor, Genèse d'un imaginaire francophone, Gallimard, 2005.

29. Discours prononcé à Lille, en février 1956 : en 1956, F. H. Boigny est alors ministre délégué à la Présidence du Conseil du gouvernement Guy Mollet; cité par Kazadi Ntole, L'Afrique afrofrancophone, Institut d'études créoles et francophones, Collection "Langues et développement ", 1991.

30. Cité par Kazadi Ntole, op. cit.

31. Du journaliste Raymond Cartier qui écrivait : «Le colonialisme a toujours été une charge en même temps qu'un profit, souvent une charge plus qu'un profit. Dans les conditions et sous les servitudes politiques actuelles, c'est plus vrai que jamais. » (Paris-Match, 18 août 1956).

32. Senghor (Léopold Sédar), « Le français, langue de culture ", art. cit.

33. Hagège (Claude), Le français, histoire d'un combat, Paris, Librairie générale française, 1998.

34. Conklin (Alice L.), A Mission to Civilize, the Republican Idea of Empire in France and West Africa, 1895-1930, Stanford University Press, Stanford, California, 1997.

35. "Le français ne vous appartient plus. Nous l'avons en partage », Le Monde, supplément « Langue française » du 27 avril 2006.

\section{RÉSUMÉS}

Cette contribution met en perspective le récit traditionnel de la Francophonie et les raisons sociologiques et économiques qui ont pu pousser les élites africaines à conserver la langue française en dépit de la décolonisation. Ce récit francophone, soutenu dans une certaine mesure par la théorie postcoloniale de l'appropriation, tend à sacraliser la langue française. Or, compte tenu des liens très forts que le récit national français entretient avec sa langue, le récit francophone a ainsi pu paradoxalement contribuer à conforter en France une mémoire partiellement positive de la mission civilisatrice.

The aim of this paper is to put into perspective the official narrative of Francophonie with the social and financial factors which may explain why African elites safeguarded the French language beyond decolonization. This francophone narrative, which is to a certain extent reinforced by postcolonial theory, completes the hagiographic French perception of the French language. Just like French national history and the French language are deeply embedded into one another, the francophone narrative has reinforced the French national narrative. As a consequence, the francophone narrative may have paradoxically strengthened a positive collective memory of the mission civilisatrice. 
INDEX

Mots-clés : francophonie, langue française, colonisation, élites francophones, nationalisme linguistique, appropriation

Keywords : francophonie, french language, colonization, francophone elites, linguistic nationalism, appropriation

\section{AUTEUR}

\section{ALICE GOHENEIX}

CEVIPOF - Centre d'études politiques de Sciences politiques

Paris, France

alice.goheneix@sciences-po.org 